\title{
(50000) Quaoar: Surface composition variability
}

\author{
M. A. Barucci ${ }^{1}$, C. M. Dalle Ore ${ }^{2,6}$, D. Perna ${ }^{1}$, D. P. Cruikshank ${ }^{2}$, A. Doressoundiram ${ }^{1}$, A. Alvarez-Candal ${ }^{3}$, \\ E. Dotto ${ }^{4}$, and C. Nitschelm ${ }^{5}$ \\ ${ }^{1}$ LESIA - Observatoire de Paris, PSL Research University, CNRS, Sorbonne Univ., UPMC Univ. Paris 06, Univ. Paris Diderot, \\ Sorbonne Paris Cité, 92195 Meudon Principal Cedex, France \\ e-mail: antonella.barucci@obspm. fr \\ 2 NASA Ames Research Center, Mail Stop 245-6, Moffett Field, CA 94035, USA \\ 3 Observatorio Nacional, Rio de Janeiro, Brazil \\ 4 INAF-Osservatorio Astronomico di Roma, 00040 Monte Porzio Catone, Roma, Italy \\ 5 Unidad de Astronomía, Facultad de Ciencias Básicas, Universidad de Antofagasta, Antofagasta, Chile \\ ${ }^{6}$ Carl Sagan Center, SETI Institute, 189 Bernardo Ave, Mountain View, CA 94043, USA
}

Received 17 March 2015 / Accepted 2 October 2015

ABSTRACT

\begin{abstract}
Aims. The goal of this work is to investigate the composition of the surface of (50000) Quaoar and its spatial variability.
Methods. We present new continuous spectra from the visible to near-IR $(0.3-2.3 \mu \mathrm{m})$ obtained with the X-Shooter instrument at the VLT-ESO at four different epochs on the surface of Quaoar. The data represent the highest spectral resolution data ever obtained for this object and the first near-IR dataset acquired in a single exposure over the entire wavelength range. They are complemented by previously published photometric observations obtained in the near-IR (3.6, $4.5 \mu \mathrm{m})$ with the Spitzer Space Telescope, which provide an extra set of constraints in the model calculation. Spectral modelling was performed for the entire wavelength range by means of a code based on the Shkuratov radiative transfer formulation and of an updated value of albedo obtained from recent Herschel observations.

Results. We obtained compositional information for different observed areas that can cover about $40 \%$ of the assumed rotational period of $8.84 \mathrm{~h}$. Our analysis helps to prove the presence of $\mathrm{CH}_{4}$ and $\mathrm{C}_{2} \mathrm{H}_{6}$, as previously reported, along with indications of the possible presence of $\mathrm{NH}_{3} \cdot \mathrm{H}_{2} \mathrm{O}$. New evidence of $\mathrm{N}_{2}$ is inferred from the shift in the $\mathrm{CH}_{4}$ bands. The albedo at the two Spitzer bands suggests there may be $\mathrm{CO}$ diluted in $\mathrm{N}_{2}$, and $\mathrm{CO}_{2}$ for one of the surface locations.

Conclusions. The spectral similarities indicate the overall homogeneity of the surface composition of one hemisphere of Quaoar, while some subtle variations are apparent when modelling. The presence of $\mathrm{NH}_{3} \cdot \mathrm{H}_{2} \mathrm{O}$ would support the idea that Quaoar's surface may be relatively young.
\end{abstract}

Key words. Kuiper belt objects: individual: (50000) Quaoar - techniques: spectroscopic

\section{Introduction}

The possibly dwarf planet (50000) Quaoar is one of the largest classical trans-Neptunian objects (TNOs) in a binary system, and it has a small satellite named Waywot with a size that is about $5 \%$ of the primary. It appears to be a particularly fascinating object in the outer solar system with an estimated density of $1.99 \pm 0.46 \mathrm{~g} \mathrm{~cm}^{-3}$ derived from the first multi-chord stellar occultations by Braga-Ribas et al. (2013), which is significantly lower than the one proposed by Fraser et al. (2013a), and it agrees with the determination by Fornasier et al. (2013) estimated on the basis of the new Herschel far-IR data.

A first estimation of its diameter of $1260 \pm 190 \mathrm{~km}$ was obtained by Brown \& Trujillo (2004) from direct imaging with the HST, while a recent determination obtained with Herschel observations together with revised Spitzer data reported a diameter of $1036 \pm 31 \mathrm{~km}$ with an albedo of $12.7 \%$ (Fornasier et al. 2013). Braga-Ribas et al. (2013) reported a preferred solution for the effective diameter of $1111 \pm 5 \mathrm{~km}$ obtained from a multichord stellar occultation. The size of (50000) Quaoar is comparable to Pluto's moon Charon.

* Based on observations made with ESO Very Large Telescope under programme ID 091.C-0057(A).
The rotational period was first estimated to be approximately $17.68 \mathrm{~h}$ by Ortiz et al. (2003) with a double-peaked lightcurve, while later a period of $8.84 \mathrm{~h}$ with single peak solution was proposed by Rabinowitz et al. (2007) and considered also possible by Thirouin et al. (2010). Several spectroscopic observations have been carried out by different authors using Subaru, Gemini, and VLT-Sinfoni (Guilbert et al. 2009). The first near-IR (NIR) observations were obtained by Jewitt \& Luu (2004), who recognized the presence of crystalline $\mathrm{H}_{2} \mathrm{O}$ and claimed the detection of a weak absorption possibly associated with ammonia hydrates. The presence of crystalline $\mathrm{H}_{2} \mathrm{O}$ ice and ammonia hydrates on a surface can be key in understanding the processes acting on it. Both are indicative of a young surface possibly refreshed by cryo-volcanic activity. The accurate fitting model by Dalle Ore et al. (2009) on high quality data reported a surface composition containing crystalline water ice, $\mathrm{CH}_{4}$, as well as possibly $\mathrm{C}_{2} \mathrm{H}_{6}$ and organic materials, products of irradiation of $\mathrm{CH}_{4}$. The band at $2.2 \mu \mathrm{m}$ was present in the observations of Dalle Ore et al. (2009) and was fitted by their model. However, it was attributed to $\mathrm{CH}_{4}$, as previously done by Schaller \& Brown (2007a), instead of ammonia hydrates. To understand the dichotomy between volatile-rich and volatile-free surfaces in the outer solar system, Schaller \& Brown (2007b) constructed a 
simple model of atmospheric escape of volatile ices over the age of the solar system. They claimed that while most TNOs are too small and warm to retain their initial volatile ices to the present day, a small number are large and cold enough to maintain these ices on their surfaces. Quaoar and a few other objects are predicted to be in the transition region where they may have lost some of their volatile components $\left(\mathrm{N}_{2}\right)$ but retained others $\left(\mathrm{CH}_{4}\right)$.

Quaoar, classified as RR type (Barucci et al. 2005) with its very red spectral slope (Fornasier et al. 2004; Alvarez-Candal et al. 2008), also appears to be rich in more complex irradiation products. Its red surface is likely due to the continued surface irradiation of $\mathrm{CH}_{4}, \mathrm{C}_{2} \mathrm{H}_{6}$, and their products. When assuming that Quaoar has a moderate obliquity, $\mathrm{CH}_{4}$ should be volatile enough to seasonally migrate, while $\mathrm{C}_{2} \mathrm{H}_{6}$ and other irradiation products would be non-volatile at Quaoar's temperature. Brown et al. (2011) hypothesize that Quaoar's red coloration could be associated with $\mathrm{CH}_{3} \mathrm{OH}$, because when irradiated, this ice is known to produce a red residue (Brunetto et al. 2006). Recently, Dalle Ore et al. (2015) have focused on "ultra-red" TNOs and Centaurs. They report that hydrocarbons and/or methanol may be present in the group considered, in support of the Brown et al. (2011) hypothesis. With their observations of Quaoar at a close passage with a background star, Fraser et al. (2013b) do not find indication of an isothermal $\mathrm{N}_{2}$ or $\mathrm{CO}$ atmosphere, but could not rule out the possibility of a tenuous $\mathrm{CH}_{4}$ atmosphere. Braga-Ribas et al. (2013) set an upper limit of about 20 nbar surface pressure for a global atmosphere of pure methane from multi-chord stellar occultations by Quaoar, but they did not exclude a local, denser atmosphere.

If we assume continuous irradiation processes on Quaoar's surface, the current presence of $\mathrm{CH}_{4}$ seems to imply that the ice is being renewed or preserved there. Otherwise, $\mathrm{CH}_{4}$ should be completely altered as proposed by Moore \& Hudson (2003) and Hudson et al. (2009). To substantiate the presence of methane on its surface and to investigate whether Quaoar is therefore likely to have an irregular covering of irradiation products, we performed new observations of different regions of the surface in spite of the difficulty in defining the different rotational longitudes due to the uncertainty of the rotational period.

In this paper we present observations obtained with a newgeneration spectrograph recording simultaneously from ultraviolet to NIR $(0.3-2.3 \mu \mathrm{m})$, optimized to detect surface variations. The data are complemented by previously published photometric observations (Dalle Ore et al. 2009) obtained in the NIR (3.6, $4.5 \mu \mathrm{m}$ ) with the Spitzer Space Telescope. Along with the updated visible albedo determined by Fornasier et al. (2013), the Spitzer data provide an extra set of constraints in the model calculation process. For each of the four spectra, we performed spectral modelling first in the entire wavelength range from 0.3 to $2.3 \mu \mathrm{m}$ by means of a code based on the Shkuratov et al. (1999) radiative transfer formulation of the slab model, then by adding the Spitzer data for additional constraints.

\section{Observations and data reduction}

Observations were performed in August 2013 at the ESO-VLT in service mode. We used the X-Shooter ${ }^{1}$ instrument, an echelle spectrograph (Vernet et al. 2011) designed to obtain a spectrum from the near-ultraviolet up to the NIR simultaneously by splitting the incoming light into three beams redirected to different

\footnotetext{
http://www.eso.org/sci/facilities/paranal/ instruments/xshooter/
}

Table 1. Observational circumstances.

\begin{tabular}{cccc}
\hline \hline Objects & Date obs. (UT) & Exptime (s) & Airmass range \\
\hline Quaoar01 & $2013-08-05 T 01: 32$ & $4 \times 900$ & $1.014-1.066$ \\
Quaoar02 & $2013-08-05 T 02: 45$ & $4 \times 900$ & $1.078-1.240$ \\
SA110361 & $2013-08-05 T 03: 56$ & $4 \times 30$ & $1.185-1.200$ \\
HD147284 & $2013-08-08 T 00: 43$ & $4 \times 3$ & $1.016-1.020$ \\
Quaoar03 & $2013-08-08 T 00: 58$ & $4 \times 900$ & $1.013-1.039$ \\
Quaoar04 & $2013-08-08 T 02: 10$ & $8 \times 600$ & $1.047-1.253$ \\
SA110361 & 2013-08-08T03:48 & $4 \times 30$ & $1.192-1.207$ \\
\hline
\end{tabular}

arms: ultraviolet-blue (UVB), visible (VIS), and NIR. We used the SLIT mode, selecting slit widths of 1.3, 1.2, and 1.2 arcsec for the UVB, VIS, and NIR arms, respectively, and nodding on the slit. Along with Quaoar, we observed the stars SA 110-361 and HD 147284, used as standard stars to remove telluric and solar signatures. Exposure times and airmasses are shown in Table 1.

All data were reduced using the pipeline provided by ESO v.2.0.0. This produces, arm by arm, master flat fields, wavelength maps, and distortion maps and combines the different exposures and sky subtraction. The final spectra were extracted using the apall task within the IRAF package. Quaoar's spectra were divided by those of SA 110-361. We note that the ratio of the two standard stars observed on August 8 is flat, and the resulting spectra were cleaned of remaining bad pixels, as well as re-binned to increase the signal-to-noise ratio $(\mathrm{S} / \mathrm{N})$. See Alvarez-Candal et al. (2011) for more details.

\section{Data analysis}

We present new visible to NIR $(0.3-2.3 \mu \mathrm{m})$ spectrophotometric data obtained with the X-Shooter instrument at the VLT-ESO facility in four different epochs on the surface of Quaoar. The newly obtained data cover about $40 \%$ of the object's rotation with the assumption that the rotational period is $8.84 \mathrm{~h}$. The observations show a strong similarity among the surface spectra, as shown in Fig. 1 (Q1-Q4 spectra). The spectra are compared with a previously obtained spectrum (Q2008 in Fig. 1) also acquired at the VLT-ESO facility, but with different instruments (FORS for the visible, ISAAC for the $J$-band, and SINFONI for the $H$ - and $K$-bands) whose spectra were adjusted by means of photometric colours (Dalle Ore et al. 2009). The advantage of the X-Shooter spectrograph is in the simultaneous spectrum acquisition from the visible to the NIR, resulting in the more accurate spectral information needed to investigate surface composition. Moreover, the resolving power of X-Shooter, almost 4000 in the NIR arm, is greater than for Sinfoni (at most 1500).

When closely inspected, new and previously analysed (Q2008) datasets show slight differences in the visible slope. The slopes of the four new observations were computed on the reflectance spectra continuum using a standard least square technique for a linear fit in the wavelength range between 0.5 and $0.8 \mu \mathrm{m}$, as described in Alvarez-Candal et al. (2008), and are reported in Table 2, along with the slope computed for the previous observation (Q2008) by Alvarez-Candal et al. (2008). Furthermore, the $\mathrm{H}_{2} \mathrm{O}$ band at $1.5-1.65 \mu \mathrm{m}$ appears to be somewhat deeper than in the new datasets, hinting at a possible misalignment of the $H$ - and $K$-bands in the Q2008 data. Consequently, a somewhat different composition is expected from modelling the new data. 
M. A. Barucci et al.: (50000) Quaoar: Surface composition variability

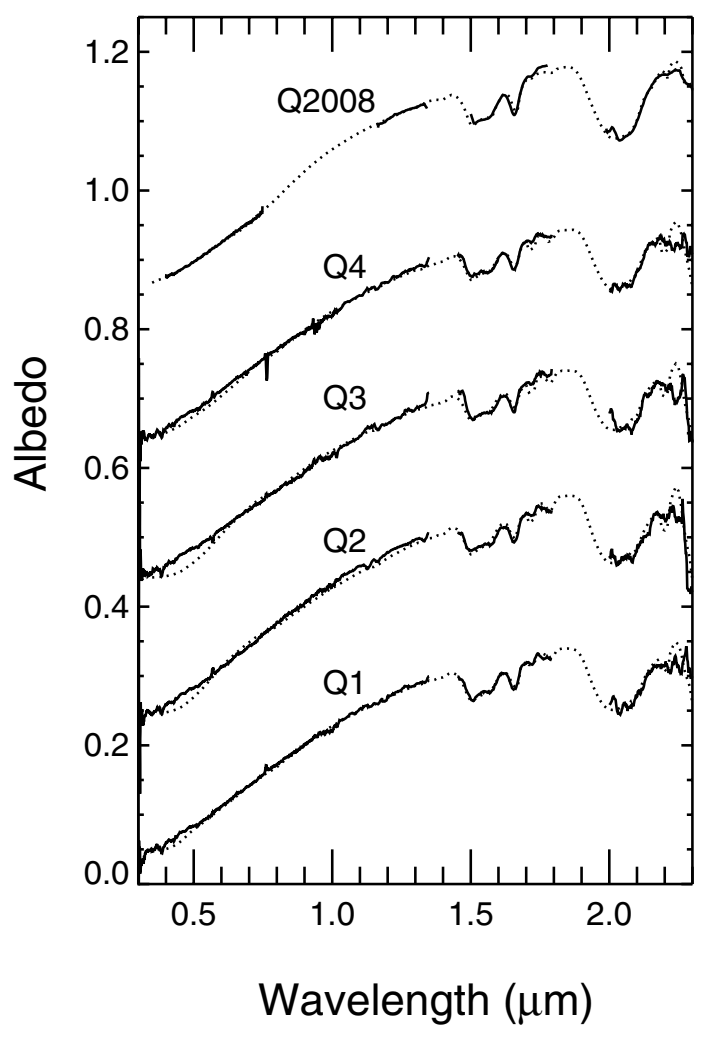

Fig. 1. Five spectra of (50000) Quaoar (solid lines) with their corresponding spectral models (dotted lines) calculated for the spectral region shown and giving most weight to the $H$-band. Spectra labelled Q1 through Q4 are newly obtained (observing circumstances are reported in Table 1), while Q2008 is the spectrum analysed in Dalle Ore et al. (2009). The spectra are represented in albedo using the value reported by Fornasier et al. (2013) with the correction as described in Sect. 3. The top four spectra have been shifted by $+0.2,+0.4,+0.6$, and +0.8 units for clarity.

The spectral data are complemented by previously published photometric observations (Dalle Ore et al. 2009) obtained in the NIR (3.6, $4.5 \mu \mathrm{m}$ - IRAC data) with the Spitzer Space Telescope, which provide an extra set of constraints in the modelling process. However, the different observing dates and the uncertainty of the rotational period preclude establishing the spatial consistency between the two sets, thus introducing an extra level of uncertainty in the modelling effort. An albedo value of $12.7 \%$ (Fornasier et al. 2013) was adopted for both spectral and photometric datasets adjusted to a value of $11.2 \%$, which corresponds to a phase angle of about $2^{\circ}$. This was done, following Dalle Ore et al. (2015), to adjust for the coherent backscattering effects (CBE) that are likely to affect Quaoar's albedo when observed at phase angles smaller than $2^{\circ}$ (Rabinowitz et al. 2007), as in the case of the adopted $\mathrm{V}$ albedo. Since the albedo level has a direct influence on the number of ices inferred by the models, taking the CBE effect into account and correcting to the proper, slightly lower albedo, increases the precision of our modelling results.

\section{Modelling}

Spectral modelling was achieved by means of a radiative transfer code based on the Shkuratov formulation of the slab approximation (Shkuratov et al. 1999). The best-fitting models described in Table 3 are intimate (salt and pepper-like) mixtures that include some molecular or intra-mixtures of tholins and ice
Table 2. Computed slopes in the visible spectra.

\begin{tabular}{lcc}
\hline \hline Objects & $S\left(\%(100 \mathrm{~nm})^{-1}\right)$ & Error \\
\hline Quaoar01 & 27.6 & \pm 0.3 \\
Quaoar02 & 27.9 & \pm 0.2 \\
Quaoar03 & 25.8 & \pm 0.1 \\
Quaoar04 & 26.1 & \pm 0.5 \\
Quaoar2008* & 28.5 & \pm 0.8 \\
\hline
\end{tabular}

Notes. ${ }^{(*)}$ The slope reported for the spectrum Q2008 is the value computed by Alvarez-Candal et al. (2008) for the visible spectrum adopted in Dalle Ore et al. (2009).

where the refractive index is calculated by means of the "effective medium" approximation (Bohren \& Huffman 1983; Wilson et al. 1994; Cuzzi \& Estrada 1998). Molecular mixing has its main effect on the visible part of the spectrum, which explains the need for more (triton) tholin in the mixtures along with the fraction contributing in the molecularly mixed part. Required input to the calculations are optical constants, the real and imaginary index of refraction of each material, the relative amounts of the components, and their grain sizes. All parameters are listed in Table 3, along with the optical constant references.

As noted, the new dataset is complemented by previously published photometric Spitzer observations, although the significantly different observing dates between the two sets introduce uncertainty into the accuracy of the conjoined data. The Spitzer set extends the wavelength range, offering further constraints for the modelling. This is particularly true for the inclusion of ices whose fundamental bands fall at wavelengths longer than $2.5 \mu \mathrm{m}$, as described in Dalle Ore et al. (2015). Therefore, to take full advantage of both datasets, we approached the modelling in two steps: a) we first modelled the spectral data giving no weight to the Spitzer photometry to determine the degree of homogeneity of Quaoar's surface; b) the second step consisted in revisiting the models including the Spitzer photometry and attempting to model the entire spectrum to determine whether other ices whose dominant signatures fall at longer wavelengths could be contributing to the spectrum while still going undetected (Dalle Ore et al. 2015). We were successful at obtaining a good fit throughout the whole spectrophotometric range for two of the four datasets, Q2 and Q4.

\subsection{Modelling of spectral data}

The main goal of this part of our analysis was to determine the amount of compositional variation among the different spectra, i.e. at different longitudes on Quaoar. For each of the four spectra, we modelled the wavelength range from 0.3 to $\sim 2.3 \mu \mathrm{m}$, giving the most weight to the $H$-band where the $\mathrm{S} / \mathrm{N}$ is highest for all four spectra. Given the large number of components present in the models, convergence to a best fit was often difficult because the root mean square (rms) for different models did not vary sufficiently. To obviate the problem when choosing between models with comparable rms, we adopted the model that yielded the best fit to the $H$-band region of the spectrum.

Table 3 lists the parameters characterizing the models that best-fitted the spectral data. The first three lines in the table list the amounts of contamination included as molecular mixtures in $\mathrm{CH}_{4}$ diluted in $\mathrm{N}_{2}$, in pure $\mathrm{CH}_{4}$ (where present), and in crystalline $\mathrm{H}_{2} \mathrm{O}$ ice. The remaining components were mixed intimately in the proportions shown in the table. The fits are shown in Fig. 1. When looking at the components, it is evident that 
Table 3. Summary of best-fitting models for Quaoar spectra observed in 2013, and the best-fitting Q2008 model (Dalle Ore et al. 2009).

\begin{tabular}{|c|c|c|c|c|c|c|c|}
\hline Components $(T)$ & Q1 & Q2 & Q3 & Q4+Spitzer ${ }^{g}$ & Q2+Spitzer & Q2008 & Ref. \\
\hline Titan tholin ${ }^{a}$ & 0.13 & 0.03 & 0.05 & 0.08 & 0.13 & 0.12 & 1 \\
\hline Triton tholin ${ }^{b}$ & 0.10 & - & - & - & - & 0.06 & 2 \\
\hline Triton tholin ${ }^{c}$ & 0.10 & 0.10 & 0.10 & 0.08 & 0.08 & 0.03 & 2 \\
\hline $\mathrm{H}_{2} \mathrm{O}$ amorph $(40 \mathrm{~K})^{d}$ & $0.02(10)$ & - & - & - & - & $0.18(1)$ & 3 \\
\hline Titan tholin ${ }^{d}$ & - & - & - & - & - & $0.13(11)$ & 1 \\
\hline $\mathrm{H}_{2} \mathrm{O}$ cryst $(40 \mathrm{~K})^{d}$ & $0.07(61)$ & $0.07(26)$ & $0.08(31)$ & $0.18(16)$ & $0.06(18)$ & $0.18(15)$ & 3 \\
\hline $\mathrm{C}_{2} \mathrm{H}_{6}$ in $\mathrm{N}_{2}^{d}$ & $0.04(20)$ & $0.02(80)$ & $0.02(80)$ & - & $0.02(31)$ & $0.04(7)^{*}$ & 8 \\
\hline Triton tholin ${ }^{d}$ & $0.39(7)$ & $0.38(10)$ & $0.39(8)$ & $0.39(8)$ & $0.36(7)$ & $0.14(7)$ & 2 \\
\hline $\mathrm{N}_{2}(36-60 \mathrm{~K})^{d}$ & $0.02(200)$ & $0.03(400)$ & $0.02(200)$ & $0.02(200)$ & $0.03(200)$ & $0.20(1610)$ & 4 \\
\hline $\mathrm{CH}_{4}(30-35 \mathrm{~K})^{d}$ & $0.09(30)$ & - & - & - & - & $0.13(9)$ & 5 \\
\hline $\mathrm{CH}_{4}$ in $\mathrm{N}_{2}^{d}$ & $0.11(70)$ & $0.24(60)$ & $0.21(60)$ & $0.15(85)$ & $0.21(80)$ & - & 8 \\
\hline $\mathrm{CO}$ in $\mathrm{N}_{2}^{d}$ & - & - & - & - & $0.05(150)$ & - & 8 \\
\hline $\mathrm{AC}^{d}$ & $0.14(20)$ & $0.11(20)$ & $0.14(20)$ & $0.14(20)$ & $0.11(20)$ & - & 6 \\
\hline $\mathrm{CO}_{2}^{d}$ & - & - & - & - & $0.04(100)$ & - & 9 \\
\hline $\mathrm{NH}_{3} \cdot \mathrm{H}_{2} \mathrm{O}^{d, e}$ & $0.12(27)$ & $0.15(30)$ & $0.14(25)$ & $0.12(20)$ & $0.12(65)$ & - & 7 \\
\hline $\mathrm{rms}^{f}$ & $7.9 \times 10^{-4}$ & $1.8 \times 10^{-3}$ & $3.1 \times 10^{-3}$ & $6.1 \times 10^{-4}$ & $1.5 \times 10^{-3}$ & $1.1 \times 10^{-4}$ & \\
\hline
\end{tabular}

Notes. ${ }^{(a)}$ Material mixed as inclusions in $\mathrm{CH}_{4}$ ice diluted in $\mathrm{N}_{2} \cdot{ }^{(b)}$ Material mixed as inclusions in pure $\mathrm{CH}_{4}$ ice. ${ }^{(c)}$ Material mixed as inclusions in crystalline $\mathrm{H}_{2} \mathrm{O}$ ice. ${ }^{(d)}$ Material mixed intimately with grain size in parenthesis. Grain size is in $\mu \mathrm{m}$ for all materials except $\mathrm{N}_{2}$, for which it is in $\mathrm{mm}$. Temperature listed after the name of the component. ${ }^{(e)}$ The adopted optical constant are for a dilution of $3 \% \mathrm{NH}_{3} \mathrm{in}_{2} \mathrm{O}$. ${ }^{(f)} \mathrm{rms}$ error of each model when compared to the data. ${ }^{(g)}$ The best-fitting model of Q4 is the same of Q4+Spitzer. ${ }^{(*)}$ In this model $\mathrm{C}_{2} \mathrm{H}_{6}$ is pure rather than diluted in $\mathrm{N}_{2}$.

References. (1) Imanaka et al. (2005); (2) Khare et al. (1994); (3) Mastrapa et al. (2009); (4) Green et al. (1991); (5) Grundy et al. (2002); (6) Rouleau \& Martin (1991); (7) Roush (Brown et al. 1988); (8) Quirico et al. (1999); (9) Hansen (1997, 2005).

the variations among the four best-fitting models are small or nonexistent for most materials. The main exception is an excess of crystalline $\mathrm{H}_{2} \mathrm{O}$ ice in $\mathrm{Q} 4$ with respect to the other models, which compensates for an apparent shortage of $\mathrm{CH}_{4}$ and $\mathrm{CH}_{4}$ diluted in $\mathrm{N}_{2}$.

Among the ices, $\mathrm{CH}_{4}, \mathrm{C}_{2} \mathrm{H}_{6}$, and $\mathrm{H}_{2} \mathrm{O}$ have all been detected previously (Schaller \& Brown 2007a,b; Jewitt \& Luu 2004). The presence of $\mathrm{N}_{2}$ had been inferred based on best-fitting models in the Spitzer bands by Dalle Ore et al. (2009). In this work $\mathrm{N}_{2}$ is not only needed to improve the fit in the NIR, but its presence is supported by the fact that $\mathrm{CH}_{4}$, and $\mathrm{C}_{2} \mathrm{H}_{6}$ show bands that are shifted by an amount corresponding to what is expected when those ices are mixed in an $\mathrm{N}_{2}$ matrix. The need for $\mathrm{CH}_{4}$ diluted in $\mathrm{N}_{2}$ is clearly shown in Fig. 2, where the dashed line, highlighting the behaviour of the pure $\mathrm{CH}_{4}$ ice, is shifted to the right of the $1.67-\mu \mathrm{m}$ band. The wavelength shift due to the $\mathrm{CH}_{4}: \mathrm{N}_{2}$ matrix effect is most clearly seen in the $1.67-\mu \mathrm{m}$ band, but the profile of this band is affected by the $1.65-\mu \mathrm{m} \mathrm{H}_{2} \mathrm{O}$ ice band. This coincidence of bands also occurs in the spectrum of Triton (Fig. 4 of Cruikshank et al. 2000), causing the centroid of the resulting band to lie at $\sim 1.657 \mu \mathrm{m}$. The centroid of the band on Quaoar is $\sim 1.654 \mu \mathrm{m}$, in satisfactory agreement with the Triton result, and thus confirms that the $\mathrm{CH}_{4}$ on Quaoar is dissolved in the $\mathrm{N}_{2}$ matrix. Figure 2 shows the Q1 best-fitting model, which includes both pure and diluted $\mathrm{CH}_{4}$. The absence of pure $\mathrm{CH}_{4}$ in the models Q2, Q3, and Q4 also agrees with the new interpretation of Pluto's near-surface ice by Trafton (2015).

The presence of $\mathrm{NH}_{3} \cdot \mathrm{H}_{2} \mathrm{O}$ is proposed here and supports the claim by Jewitt \& Luu (2004), that the presence of ammonia hydrates explains a feature at $\sim 2.2 \mu \mathrm{m}$. The same band was later attributed to $\mathrm{CH}_{4}$ by Schaller \& Brown (2007a,b). The $\mathrm{NH}_{3} \cdot \mathrm{H}_{2} \mathrm{O}$ presence is now deduced not only from the weak band at $2.21 \mu \mathrm{m}$ but also from the relative strength of the 2.0and $1.5-\mu \mathrm{m} \mathrm{H}_{2} \mathrm{O}$ bands. If only $\mathrm{H}_{2} \mathrm{O}$ is included in the models and the model is optimized to fit the $1.5-\mu \mathrm{m}$ band, then the

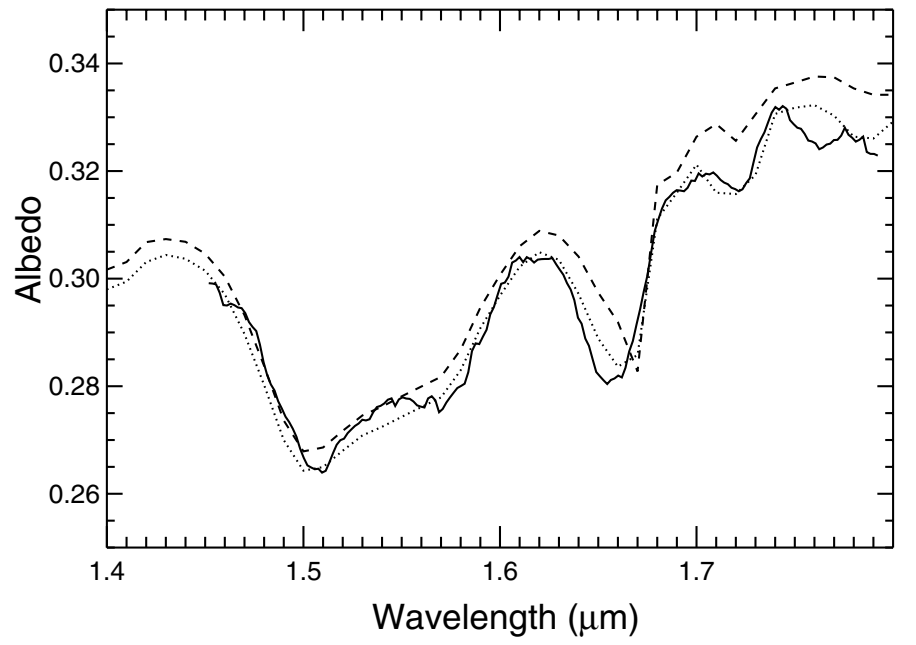

Fig. 2. Spectrum zoom of (50000) Quaoar (Q1, solid line) and corresponding best-fitting spectral model (dotted line, described in Table 3) and equivalent model calculated with pure $\mathrm{CH}_{4}$ alone (dashed line).

corresponding 2.0- $\mu \mathrm{m}$ band is too shallow compared to the observations shown in Fig. 3. The addition of $\mathrm{NH}_{3} \cdot \mathrm{H}_{2} \mathrm{O}$ in the amounts shown in Table 3 brings the depth of the 2.0- $\mu \mathrm{m}$ band to fit the data. However, we should also note that some part of the discrepancy in depth between the two $\mathrm{H}_{2} \mathrm{O}$ bands could be caused by the contribution of sub-micron particles introducing a Rayleigh scattering component to the spectrum (Clark et al. 2012). Such a component would be missing from our model as the adopted modeling code does not include a Rayleigh scattering module and might introduce the observed mismatch between the model and the observations.

A small amount of amorphous $\mathrm{H}_{2} \mathrm{O}$ ice is present in the bestfitting model of one of the four new datasets (Q1). Amorphous 


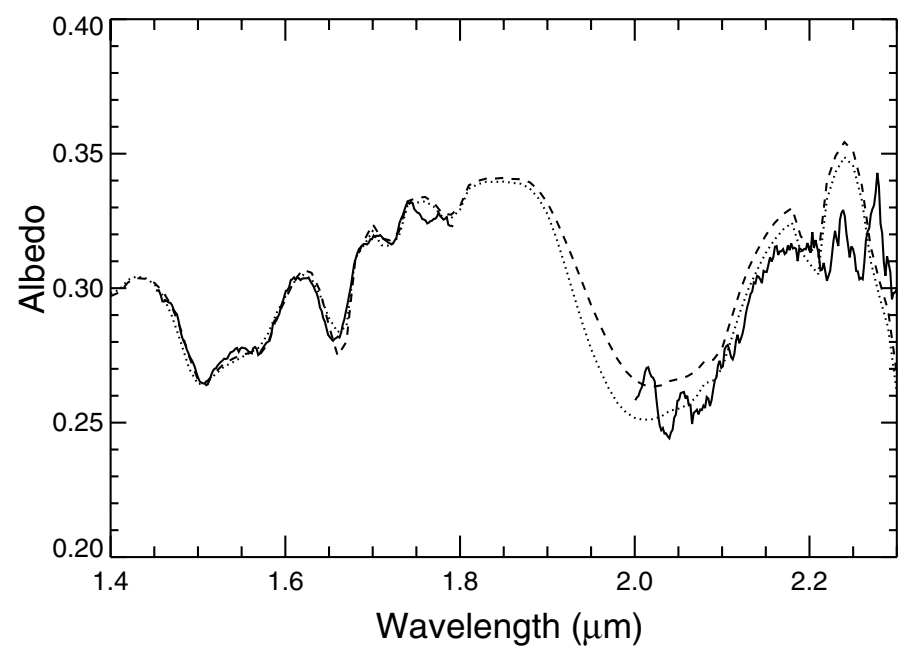

Fig. 3. Spectrum zoom of (50000) Quaoar (Q1, solid line) and corresponding best-fitting spectral model (dotted line, described in Table 3) and equivalent model calculated without $\mathrm{NH}_{3} \cdot \mathrm{H}_{2} \mathrm{O}$ (dashed line). The lack of $\mathrm{NH}_{3} \cdot \mathrm{H}_{2} \mathrm{O}$ prevents the model from properly fitting the bottom of the $2-\mu \mathrm{m}$ band.

$\mathrm{H}_{2} \mathrm{O}$ is expected to be present on the surface of bodies that have been exposed to radiation or particle bombardment, causing the change from crystalline to amorphous ice in a timeframe of $\sim 10^{7}$ yr (Jewitt \& Luu 2004; Cooper et al. 2003; Mastrapa et al. 2013). The predominance of crystalline ice might therefore indicate the relatively young age of Quaoar's surface, as previously suggested by Jewitt \& Luu (2004). This is supported by the presence of $\mathrm{NH}_{3} \cdot \mathrm{H}_{2} \mathrm{O}$ which is also unstable so possibly a young component of the surface. The crystalline $\mathrm{H}_{2} \mathrm{O}$ ice and ammonia hydrates detected in the case of Charon allowed Cook et al. (2007) to argue that surface renewal is needed.

Predicted for objects with red spectra (Brown et al. 2011), $\mathrm{CH}_{3} \mathrm{OH}$ is not found in any of the four spectra, implying that the presence of this ice is not a necessary condition for reddening TNO's surfaces. Other ices, in particular $\mathrm{CH}_{4}$ (Brunetto et al. 2006), have been shown to be plausible precursors for reddening of these surfaces, as also noticed by Dalle Ore et al. (2015).

\subsection{Modelling spectral and Spitzer data}

Because of the relatively small variations in surface composition obtained from the spectral fitting, we made use of the Spitzer photometry in conjunction with spectral data not necessarily from the same geographical region. The limitation of this approximation is further emphasized by the fact that the Spitzer data taken in two different epochs show a discrepancy in the $4.5-\mu \mathrm{m}$ band. Keeping this in mind, the spectral data were given more weight in the model calculations than the Spitzer data, and of the four sets of observations, only two, Q2 and Q4, could be fitted with models that were a good match to both the spectrum and the Spitzer bands. The Spitzer photometry consists of two sets of observations taken at slightly different times to cover different parts of the surface. However, based on the hypothesis that the surface is to some extent homogeneous and on the fact that the uncertainty in the rotational period precludes the longitudinal placement of the different observations, we adopted the average of the two observations for each band. In the case of Q4, the best-fitting model to the spectral region matches the Spitzer bands with no need of additional components; its parameters are listed in Table 3 in the Q4 column. Instead, for Q2,

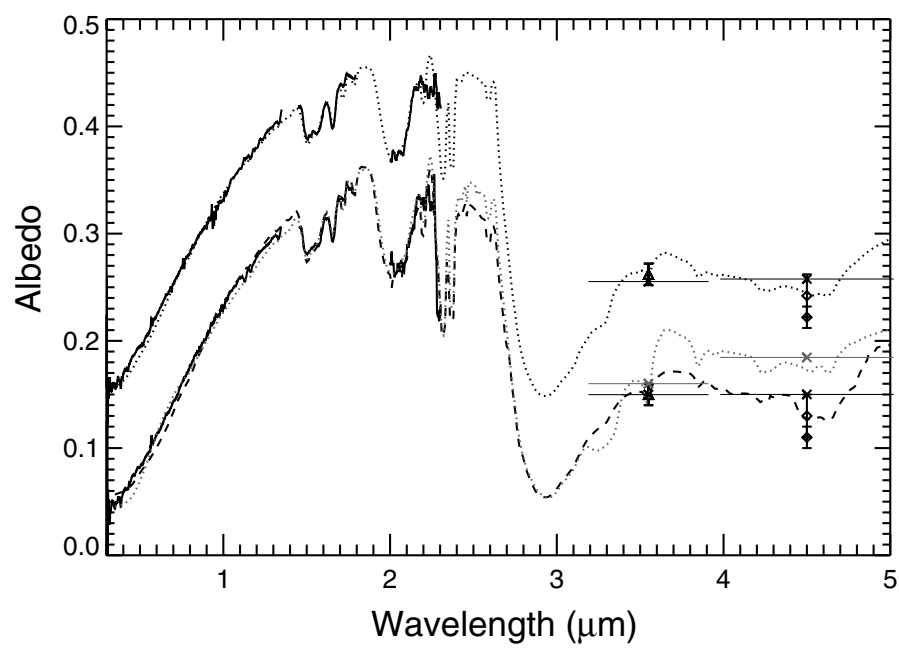

Fig. 4. Bottom spectrum: (50000) Quaoar (Q2, solid line) with Spitzer photometry data (triangle and diamond symbols) obtained at two different epochs plotted with the corresponding best-fitting spectral model (dashed line, parameters are listed in Table 3, column Spitzer) that includes $\mathrm{CO}$ diluted in $\mathrm{N}_{2}$ and $\mathrm{CO}_{2}$. The best-fitting model for the spectral dataset without $\mathrm{CO}$ and $\mathrm{CO}_{2}$ is also overlapped and shown for comparison (grey dotted line, parameters are listed in Table 3, column Q2). Both models, convolved by the Spitzer IRAC filter bandpasses (horizontal segments), are shown as crosses (black and grey, respectively). Top spectrum: (50000) Quaoar (Q4, solid line) and corresponding bestfitting spectral model (dotted line, parameters are listed in Table 3, column Q4). The model, convolved by the Spitzer IRAC filter bandpasses (horizontal segments), is shown as black crosses. The top spectrum has been shifted by +0.1 units for clarity.

the best fit was achieved with the addition of $\mathrm{CO}$ and $\mathrm{CO}_{2}$ ice, as shown in Table 3 under the Spitzer column. The best-fitting models to the entire wavelength range are shown in Fig. 4, where the fit to Q2 is shown with the one to Q4. The models were convolved to the instrumental profiles of the IRAC bands and are shown in the figure as crosses embedded in a horizontal line representing the bandpass. While the introduction of $\mathrm{CO}$ in $\mathrm{N}_{2}$ and of $\mathrm{CO}_{2}$, whose contributions darken the albedo at the second Spitzer band, helps to achieve a good fit, it is not sufficient to determine whether these ices are indeed present on the surface of Quaoar. This is due to the uncertainties in matching the spectra to the IRAC bands, as well as to the fact that, unfortunately, both $\mathrm{CO}_{2}$ and $\mathrm{CO}$ bands - both shifted and unshifted - fall in the noisy part of the new spectral data and therefore do not allow for a definitive detection and determination of their presence. In the calculations, we adopted $\mathrm{CO}$ in $\mathrm{N}_{2}$, but we do not have any evidence of the diluted versus the pure CO. The amounts listed in Table 3 for these ices should be considered as upper limits determined by their contribution to the model. A better signal in the $\sim 2 \mu \mathrm{m}$ region is needed to assess the presence of these ices.

\subsection{Comparison with previously published model}

To properly compare the models, we should first summarize the differences between the old dataset and the new. The old data were taken with different instruments and had to be scaled to the proper albedos by means of photometric measurements. The new data are instead observed as a single spectral unit. Another important difference is in the $\mathrm{V}$ albedo adopted for the old and new datasets. The old albedo was higher (19.9\%) than the currently adopted one (12.7\% adjusted for phase angle consistency with the data to $11.2 \%$ ). Also, the slope of the old data was slightly 
steeper than those of the new dataset. The greater resolving power of X-Shooter compared to Sinfoni allows us to detect the wavelength shifts in the $\mathrm{CH}_{4}$ bands better because the bottom of the absorption bands are better resolved.

The previously published Quaoar model by Dalle Ore et al. (2009) is listed in Table 3 (column Q2008) along with the models that fit the new datasets best. A comparison among the models shows a few differences that can, however, be explained to some degree. When inspecting the table from the top, the first noticeable discrepancy is in the relative amounts of tholins mixed with $\mathrm{CH}_{4}$ and $\mathrm{H}_{2} \mathrm{O}$. Compared to the new models, the old one includes a smaller relative amount of this red component. However, the amounts of $\mathrm{CH}_{4}$ and $\mathrm{H}_{2} \mathrm{O}$ are larger in the old model, therefore ultimately supplying more reddening material to the surface. The amount of triton tholin mixed intimately is also smaller in the Q2008 model, but titan tholin also contributes, although it is not present in the new models.

The next significant discrepancy is in the amount and grain size of amorphous $\mathrm{H}_{2} \mathrm{O}$ ice included in the Q2008 model. The ice was included to help fit the Spitzer bands, but a non-physical small grain size had to be adopted to achieve the goal. Dalle Ore et al. (2009) conclude that the surface composition might still be missing some components. However, while we had tested $\mathrm{CO}$ and $\mathrm{N}_{2}$, which helped but not enough, we had not considered $\mathrm{CO}_{2}$. In our current model of Q2 fitting the Spitzer bands (Table 3, Spitzer column), we achieved the desired fit with acceptable grain sizes by including all three ices. This could be considered as further evidence of these ices on Quaoar. Furthermore, the significantly larger amount of $\mathrm{N}_{2}$ adopted in the Q2008 model than in the new models can also be justified because of the need to fit the Spitzer bands as stated in the original paper.

The different (larger) amounts of crystalline $\mathrm{H}_{2} \mathrm{O}$ in the Q2008 model can be justified by the previously adopted higher $\mathrm{V}$ albedo requiring more ices to fit the overall albedo level. $\mathrm{CH}_{4}$ was also higher in the old model, but when both pure and $\mathrm{N}_{2}$ diluted $\mathrm{CH}_{4}$ are considered the new models are not very different from the old. $\mathrm{C}_{2} \mathrm{H}_{6}$ was included pure in the old model and is diluted in $\mathrm{N}_{2}$ in the new models. The $\mathrm{S} / \mathrm{N}$ of the new data is unfortunately not sufficient to establish whether this is the correct $\mathrm{C}_{2} \mathrm{H}_{6}$ form. We adopted it more for consistency with the other ices than as a detection. The amounts among the models are consistent.

Lack of amorphous carbon (AC) in the Q2008 model is probably due to the difference in $\mathrm{V}$ albedo, which is much darker in the new dataset. Finally the addition of $\mathrm{NH}_{3} \cdot \mathrm{H}_{2} \mathrm{O}$ to the new models reflects the importance of the entire spectrum being observed simultaneously. The misalignment of the $H$ - and $K$-band precluded any inference as to the presence of $\mathrm{NH}_{3} \cdot \mathrm{H}_{2} \mathrm{O}$ in the old dataset.

\section{Discussion and conclusions}

New simultaneous spectra from the visible to NIR $(0.3-2.3 \mu \mathrm{m})$ obtained with the X-Shooter instrument at the VLT-ESO have been presented, along with models that describe the surface of Quaoar. The observations were taken at four different longitudes covering about $40 \%$ of Quaoar's rotation (in the hypothesis of a single peak period of $8.84 \mathrm{~h}$ ). The spectra are very consistent, which implies homogeneity of the surface composition on one hemisphere of Quaoar. The homogeneity is also supported to some degree by the best-fitting models calculated with a radiative transfer code. However, the newly obtained spectra show some discrepancy with the 2008 observation of Quaoar, shown as Q2008 in Fig. 1, mostly in the shape of the slope in the visible and with a deeper band at $1.5-1.65 \mu \mathrm{m}$. The uncertainty of the rotational period does not allow the corresponding longitude of the spectrum Q2008 to be determined unlike for the new observations. Therefore it is possible that the spectral variations could be because we sampled different terrains but also because the 2008 observations were not obtained simultaneously with three different instruments.

A detailed comparison of compositions deduced from modelling the Q2008, as well as models of the four new spectra seems to indicate a fairly consistent composition with a few justifiable exceptions, further supporting the idea of a homogeneous surface. Prompted by this result, we proceeded to include the Spitzer IRAC data previously published by Dalle Ore et al. (2009), with the new spectral data used to constrain the composition of the surface better. New indications of the presence of $\mathrm{N}_{2}$ on Quaoar's surface were inferred from the shifts of the $\mathrm{CH}_{4}$ bands, as expected when this material is embedded in an $\mathrm{N}_{2}$ matrix. $\mathrm{CO}$ in $\mathrm{N}_{2}$ is only an upper limit since its bands are hardly visible in a part of the spectrum where the $\mathrm{S} / \mathrm{N}$ is poor. Also, while we adopted $\mathrm{CO}$ in $\mathrm{N}_{2}$ for our calculations, we have no evidence that it is indeed the diluted versus the pure $\mathrm{CO}$ contributing to the albedo at the Spitzer wavelengths. However, the shifted bands of $\mathrm{CH}_{4}$, in particular, and possibly $\mathrm{C}_{2} \mathrm{H}_{6}$ are clearly aligned with those of ices diluted in $\mathrm{N}_{2}$. Pure $\mathrm{CH}_{4}$ bands are also present in one spectrum. The coexistence of pure $\mathrm{CH}_{4}$ and $\mathrm{CH}_{4}$ diluted in $\mathrm{N}_{2}$ make Quaoar closer to Pluto than originally thought. Like $\mathrm{CO}$ in $\mathrm{N}_{2}, \mathrm{CO}_{2}$ is also included in the model only as an upper limit to match the data at the two Spitzer bands. The comparison between the old Q2008 model and the new ones offers further evidence of the presence of $\mathrm{CO}$, either diluted or pure, and $\mathrm{CO}_{2}$ on Quaoar. In the old model, very small $\mathrm{H}_{2} \mathrm{O}$ ice grains had to be adopted to fit the Spitzer data. With the inclusion of $\mathrm{CO}$ in $\mathrm{N}_{2}$ and $\mathrm{CO}_{2}$ this is not necessary in the new model.

The confirmed presence of crystalline $\mathrm{H}_{2} \mathrm{O}$ ice and its abundance over the amorphous $\mathrm{H}_{2} \mathrm{O}$ ice on this object may indicate that our current understanding of the physics of the crystalline/amorphous phase transition may not be complete. The ices detected on the surface of Quaoar may suggest that there is an efficient renewal mechanism on its surface. Moreover, the concurrent presence of $\mathrm{NH}_{3} \cdot \mathrm{H}_{2} \mathrm{O}$, also a very unstable ice on the surface of TNOs, could imply that Quaoar's surface might be relatively young, as previously suggested by Jewitt \& Luu (2004). Shuchuko et al. (2014) describe the thermal evolution of TNOs on the basis of their numerical simulations as a function of the intensity of radiogenic heat and they predict the possible presence of cryovolcanism on Quaoar. Cook et al. (2007) analysed possible mechanisms for surface renewal and also conclude that the presence of crystalline $\mathrm{H}_{2} \mathrm{O}$ ice and ammonia hydrates on the surface of Charon might be evidence of cryovolcanism. Nevertheless, crystalline $\mathrm{H}_{2} \mathrm{O}$ ice has been observed around young forming stars (Van Dishoeck 2004) and amply discussed by Suh \& Kwon (2013) and Dartois et al. (2015). Consequently, we cannot argue that the mere presence of crystalline $\mathrm{H}_{2} \mathrm{O}$ ice implies cryovolcanism. However, the suggested presence of $\mathrm{NH}_{3} \cdot \mathrm{H}_{2} \mathrm{O}$ along with Quaoar's size hints at the possibility that volcanism could occur on its surface, as has been described for volatile-rich objects (Stevenson 2004). Alternatively, crystalline $\mathrm{H}_{2} \mathrm{O}$ ice could be the product of impact gardening as proposed by Porter et al. (2010) and $\mathrm{NH}_{3} \cdot \mathrm{H}_{2} \mathrm{O}$ could be seeping up to the surface of Quaoar by a diffusive mechanism, or its spectral signature could be influenced by the presence of sub-micron particles on the surface, as described by Clark et al. (2012). 
Finally, the spectrum of Quaoar is consistent with that of a cold object slowly losing the last of its volatile ices by escape in a tenuous atmosphere.

Acknowledgements. Based on observations carried out at the European Southern Observatory (ESO), Chile (programme 091.C-0057). The project is supported by the French Planetology National Programme (INSU-PNP). C.M.D.O. acknowledges support from the Outer Planets Research grant NASA NNX12AM75G. A.A.C. acknowledges financial support from $\mathrm{CNPq}$ and FAPERJ through diverse grants.

\section{References}

Alvarez-Candal, A., Fornasier, S., Barucci, M. A., et al. 2008, A\&A, 487, 741 Alvarez-Candal, A., Pinilla-Alonso, N., Licandro, J., et al. 2011, A\&A, 532, A130

Barucci, M. A., Belskaya, I. Fulchignoni, M., et al. 2005, AJ, 130, 1291

Bohren, C. F., \& Huffman, D. R. 1983, Absorption and Scattering of Light by Small Particles (New York: Wiley)

Braga Ribas, F., Sicardy, B., Ortiz, J. L., et al. 2013, ApJ, 773, 26

Brown, M. E., \& Trujillo, C. A. 2004, AJ, 127, 2413

Brown, R. H., Cruikshank, D. P., Tokunaga, A. T., et al. 1988, Icarus, 74, 262

Brown, M. E., Shaller, E. L., \& Fraser, W. C. 2011, ApJ, 739, L60

Brunetto, R., Barucci, M. A., Dotto, E., \& Strazulla, G. 2006, ApJ, 644, 646

Clark, R. N., Cruikshank, D. P., Jaumann, R., et al. 2012, Icarus, 218, 831

Cook, J. C., Steven, J. D., Roush, T. L., et al. 2007, ApJ, 633, 1406

Cooper, J. F., Christian, E. R., Richardson, J. D., \& Wang, C. P. 2003, Earth Moon Planets, 92, 261

Cruikshank, D. P., Schmitt, B., Roush, T. L., et al. 2000, Icarus, 147, 309

Cuzzi, J. N., \& Estrada, P. R. 1998, Icarus, 132, 1

Dalle Ore, C. M., Barucci, M. A., Emery, J. P., et al. 2009, A\&A, 501, 349

Dalle Ore, C. M., Barucci, M. A., Emery, J. P., et al. 2015, Icarus, 252, 31

Dartois, E., Auge, B., Brunetto, R., et al. 2015, A\&A, 576, A125

Fraser, W. C., Batygin, K., Brown, M. E., et al. 2013a, Icarus, 222, 357
Fraser, W. C., Trujillo, C., Stephens, A. W., et al. 2013b, ApJ, 774, L18

Fornasier, S., Doressoundiram, A., Tozzi, G. P., et al. 2004, A\&A, 421, 353

Fornasier, S., Lellouch, E., Muller, T., et al. 2013, A\&A, 555, A15

Green, J. R., Brown, R. H., Cruikshank, D. P., \& Anicich, V. 1991, BAAS, 23 1208

Grundy, W. M., Schmitt, B., \& Quirico, E. 2002, Icarus, 155, 486

Guilbert, A., Alvarez-Candal, A., Merlin, F., et al. 2009, Icarus, 201, 272

Hansen, G. B. 1997, Adv. S.R., 20, 1613

Hansen, G. B. 2005, J. Geophys. Res., 110, E11003

Hudson, R. L., Moore, M. H., Raines, L. L., et al. 2009, Icarus, 203, 677

Imanaka, H., Khare, B. N., McKay, C. P., \& Cruikshank, D. P. 2005, BAAS, 37 , 772

Jewitt, D. C., \& Luu, J. 2004, Nature, 432, 731

Khare, B. N., Sagan, C., Heinrich, M., et al. 1994, BAAS, 26, 1176

Mastrapa, R. M. E., Sandford, S. A., Roush, T. L., et al. 2009, ApJ, 701, 1347

Mastrapa, R. M. E., Grundy, W. M., \& Gudipati, M. S. 2013, Amorphous and Crystalline $\mathrm{H}_{2} \mathrm{O}$-Ice, The Science of Solar System Ices, 371

Moore, M. H., \& Hudson, R. L. 2003, Icarus, 161, 486

Ortiz, J. L., Gutierez, P. J., Sota, A., et al. 2003, A\&A, 409, L13

Porter, S. B., Desch, S. J., \& Cook, J. C. 2010, Icarus, 208, 492

Quirico, E., Doute, S., Schmitt, B., et al. 1999, Icarus, 139, 159

Rabinowitz, D. L., Schafer, B. E., \& Tourtellotte, S. W. 2007, AJ, 133, 26

Rouleau, F., \& Martin, P. G. 1991, ApJ, 377, 526

Schaller, E. L., \& Brown, M. E. 2007a, ApJ, 670, L49

Schaller, E. L., \& Brown, M. E. 2007b, ApJ, 659, L61

Shkuratov, Y., Starukhina, L., Hoffmann, H., \& Arnold, G. 1999, Icarus, 137, 235

Shchuko, O. B., Shchuko, S. D., Kartashov, D. V., \& Orosei, R. 2014, Planet. Space Sci., 104, 147

Suh, K.-W., \& Kwon, Y.-J. 2013, ApJ, 762, 113

Stevenson, D. J. 2004, Nature, 432, 681

Thirouin, A., Ortiz, J., Dufard, R., et al. 2010, A\&A, 522, A93

Trafton, L. M. 2015, Icarus, 246, 197

Van Dishoeck, E. F. 2004, ARA\&A, 42, 119

Vernet, J., Dekker, H., D’Odorico, S., et al. 2011, A\&A, 536, A105

Wilson, P. D., Sagan, C., \& Thompson, W. R. 1994, Icarus, 107, 288 